\title{
Valence Force Constants of N-Benzylideneanilines
}

\author{
K. Figueroa A., R. Peña C. ${ }^{+}$, and M. M. Campos-Vallette* \\ Department of Chemistry, Faculty of Sciences, University of Chile, Casilla 653, Santiago, Chile \\ + Universidad Metropolitana de Ciencias de la Educación, Dept. of Chemistry, \\ Macul 774, Santiago, Chile
}

Z. Naturforsch. 44b, 923-927 (1989); received October 20, 1988

Valence Force Constants, N-Benzylideneanilines

Valence force constants for $\mathrm{N}$-benzylideneaniline and for two $p, p^{\prime}$-disubstituted derivatives were estimated by means of a point-charge model using CNDO data. A vibrational assignment of bands which are sensitive to the electron donor-acceptor characteristic of substituents is proposed. The conformational changes are interpreted in terms of an intramolecular energy transfer between substituents.

\section{Introduction}

Within the familiy of organic compounds with $\pi$-systems, N-benzylideneaniline (NBA) is interesting because of the nature of the phenyl-N, N=C and C-phenyl bonds, and the influence of the $p, p^{\prime}$ substituents on the molecular conformation (see Fig. 1). As a consequence of the electron donor and acceptor ability of the substituents, N-benzylideneanilines adopt different conformations. Although several papers dealing with this subject have been published [1], to our knowledge, there is no information about vibrational studies on $\mathrm{N}$-benzylideneaniline (1), $p$-methylbenzylidene- $p$-nitroaniline (2) and $p$-dimethylaminobenzylidene- $p$-nitroaniline (3) compounds. The aim of the present paper is to perform a normal coordinate analysis of $\mathbf{1}$ by calculating the valence force constants in the series and then, to interpretate the influence of the substituents on the molecular conformation.

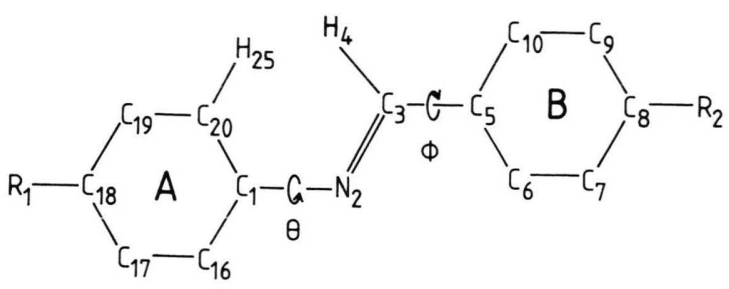

Fig. 1. $p, p^{\prime}$-Substituted N-benzylideneanilines.
1: $\mathrm{R}_{1}=\mathrm{R}_{2}=\mathrm{H}$
$\theta=55^{\circ}, \quad \varphi=10^{\circ}$.
2: $\mathrm{R}_{1}=\mathrm{NO}_{2}, \mathrm{R}_{2}=\mathrm{CH}_{3}$,
$\theta=52^{\circ}$,
$\varphi=8^{\circ}$.
3: $\mathrm{R}_{1}=\mathrm{NO}_{2}, \mathrm{R}_{2}=\mathrm{N}\left(\mathrm{CH}_{3}\right)_{2}, \quad \theta=49^{\circ}, \quad \varphi=7.1^{\circ}$.

$\mathrm{A}$ : aniline ring; $\mathrm{B}$ : benzylidene ring.

* Reprint requests to Dr. M. M. Campos-Vallette.

Verlag der Zeitschrift für Naturforschung, D-7400 Tübingen 0932-0776/89/0800-0923/\$ 01.00/0
In order to analyze the substituent effects on the conformation of N-benzylideneanilines $\mathbf{1}$ to $\mathbf{3}$ we used a point-charge model proposed by Kosmus [2]. This formulas which consist in a simple method to discuss bond properties at the equilibrium position, allows us to estimate the electronic contributions to the force constants. These valence force constants have been shown to be appropriate to describe several molecular systems [3].

\section{Experimental}

All compounds, synthesized by standard methods [4], were purified by recrystallization before measurements. Infrared spectra (IR) were recorded on a Perkin-Elmer 621 spectrophotometer for the crystal dispersed in $\mathrm{KBr}$ pellets. Raman spectra were recorded on a Coderg T 800 spectrophotometer using the $647.1 \mathrm{~nm}$ exciting line of an Spectra Physics $\mathrm{Kr}^{+}$ laser. The spectra are displayed in Fig. 2.

\section{Computational Method}

A CNDO/2 computational programme [5] was employed and experimental geometries were obtained from the literature $[6,7]$. X-ray structure determinations of compounds $\mathbf{1}$ to $\mathbf{3}$ indicate that the aniline and benzylidene rings are twisted out of the $\mathrm{C}_{1}-\mathrm{N}_{2}=\mathrm{C}_{3}-\mathrm{C}_{5}$ plane by varying angles in opposite directions (see Fig. 1). The torsion angles are defined as $\theta=\mathrm{C}_{3} \mathrm{~N}_{2} \mathrm{C}_{1} \mathrm{C}_{20}$ and $\varphi=\mathrm{N}_{2} \mathrm{C}_{3} \mathrm{C}_{5} \mathrm{C}_{6}$.

\section{Results and Discussion}

The CNDO calculations can give some insight into the electron distributions and $\sigma$ and $\pi$ polarities of bonds. Using the Wiberg bond index (W) criterium, we have a pure covalent bond if $\mathrm{W}$ equals 1 and a 


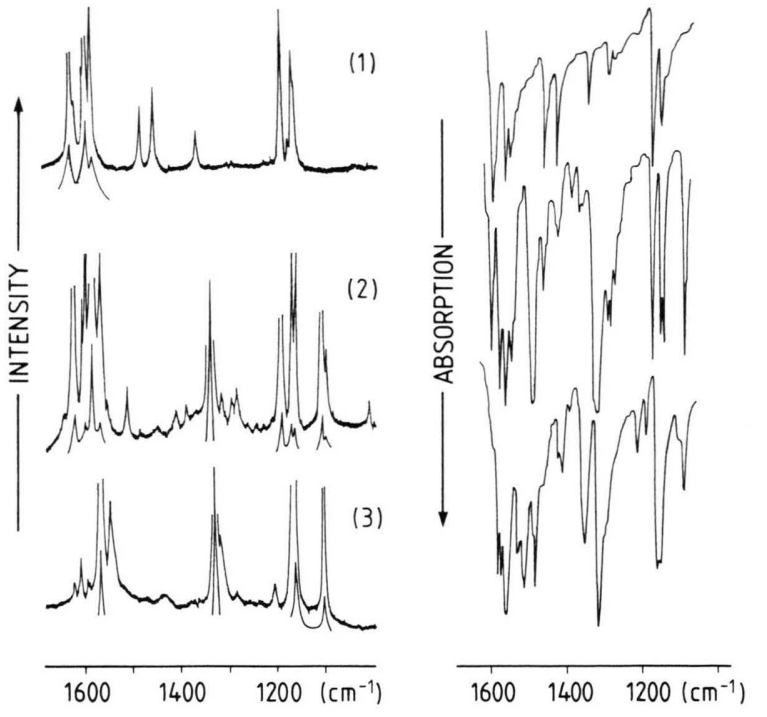

Fig. 2. Raman and Infrared spectra of N-benzylideneaniline (1), $p$-methylbenzylidene-p-nitroaniline (2) and $p$-dimethylaminobenzylidene- $p$-nitroaniline (3) in the region $1650-1100 \mathrm{~cm}^{-1}$. partial polarized bond if $\mathrm{W}$ is less than 1 [8]. Thus, we have two figures to characterize a bond: the bond index which represents the occupation of the covalent part of a bond and the atomic net charges $(\mathrm{Q})$ which show the polarities within the whole molecule. The pure covalent bonds are highly directional, or rigid, with a large force constant, in comparison to polar or ionic bonds which become more flexible with increasing ionicity.

Chemical bonds with a large force constant value are characterized by a relatively high vibrational frequency and can be regarded as rigid chemical bonds [9]. The force constants (f) for the most relevant bonds in compounds $\mathbf{1}, \mathbf{2}$ and $\mathbf{3}$ are displayed in Table I. The range of the calculated constants agrees with collected approximated numerical values for stretching forces [10]. In general, for $\mathrm{N}-\mathrm{C}$ and $\mathrm{C}-\mathrm{C}$ bonds treated as pseudodiatomics, three characteristically distinct potential wells are commonly found and may be identified with a) single bonds with $f$ close to 5.3 and 4.5 mdyn $\AA^{-1}$, b) double bonds with

Table I. Length (R) $(\AA)$, atomic net changes (Q), Wiberg indices (W), and valence force constants (f) (mdyn $\left.\AA^{-1}\right)$ for different bonds in N-benzylideneaniline (1), $p$-methylbenzylidene- $p$-nitroaniline (2) and $p$-dimethylaminobenzylidene$p$-nitroaniline $(\mathbf{3})$.

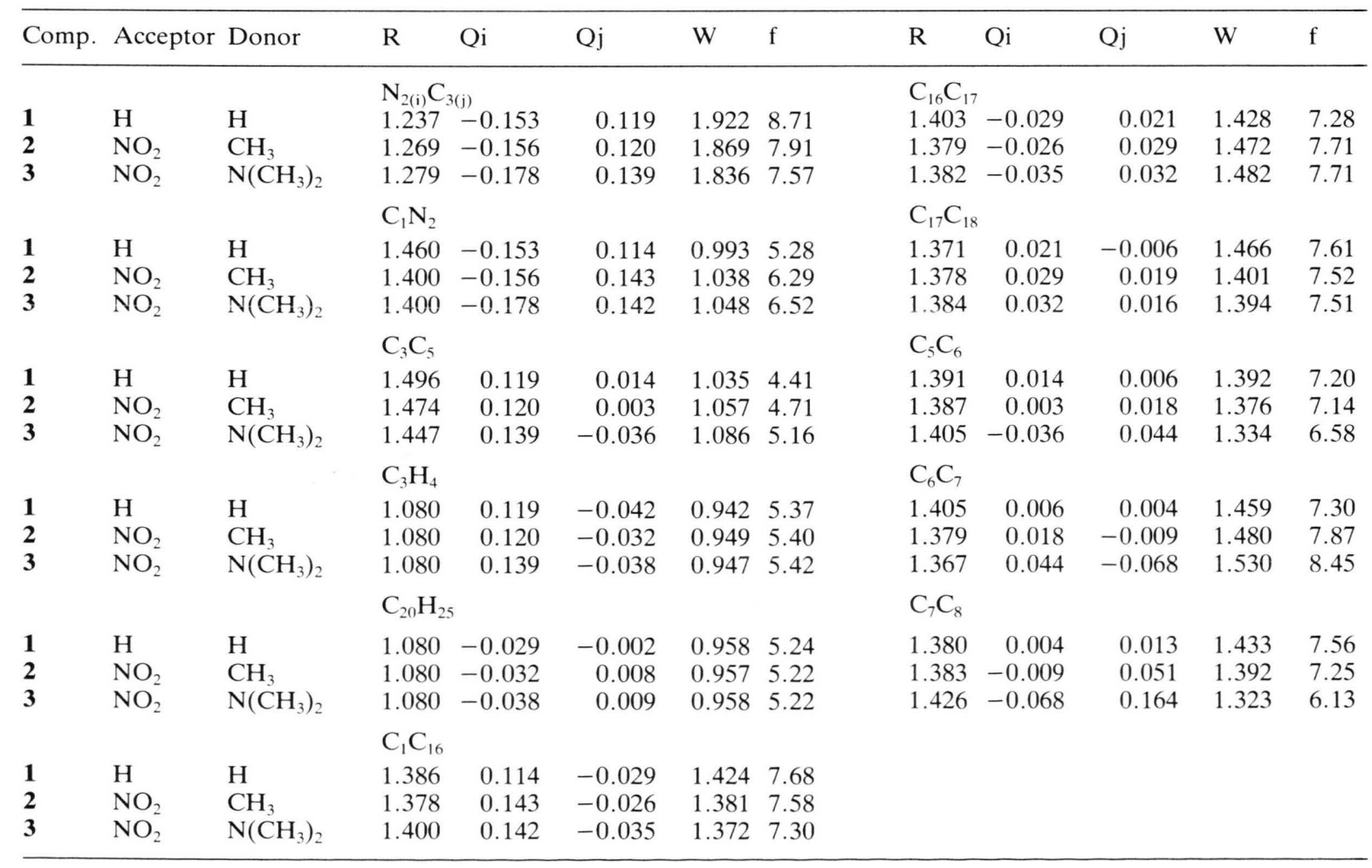


$\mathrm{f} \approx 10.5$ and 11.0 mdyn $\AA^{-1}$ and c) triple bonds with $\mathrm{f} \approx 17.0$ and 15.0 mdyn $\AA^{-1}$, respectively. The force constants are consistent with the conventional idea of single, double and triple $\mathrm{CN}$ and $\mathrm{CC}$ bonds and such bonds are always found to be associated with potential wells with force constants close to those given above. However, any observed intermediate value of $f$ may also be identified with a bond of intermediate character.

\section{$\mathrm{N}_{2} \mathrm{C}_{3}$ bonds}

Polarity changes observed in $\mathrm{N}_{2} \mathrm{C}_{3}$ bonds may be explained on the basis of both the bond lengths and the net charges of atoms directly involved (see Table I). In fact, as expected, the longer the bond distance the bigger is the resulting dipole moment of the bond. The Wiberg values suggest that the $\pi$ overlapping on these bonds decreases when increasing the relative electron donor and acceptor ability of substituents. Therefore, we should expect a similar effect on the $\mathrm{N}_{2} \mathrm{C}_{3}$ force constants (see Table I), or a frequency shifting of the corresponding stretching mode toward lower energies. In fact, this trend is verified in the IR and Raman spectra where the observed band at $1625 \mathrm{~cm}^{-1}$ in NBA is shifted to 1620 and $1575 \mathrm{~cm}^{-1}$ in compounds $\mathbf{2}$ and 3, respectively (see Fig. 2). This assignment is in agreement with reported data on Schiff's bases [11].

\section{$C_{1} N_{2}$ bonds}

In Table I it may be seen that the $\mathrm{C}_{1} \mathrm{~N}_{2}$ bond distances decreases when increasing only the acceptor property of $p$-substituents, but the bond polarity increases in the same order. In such a case it seems that the charge redistribution has a bigger influence than the variation of bond lengths. That is, the charge distribution induced by the variation of the distance makes that overall an increasing of the dipole moment is observed.

As suggested by the Wiberg indices analysis, the $\mathrm{C}_{1} \mathrm{~N}_{2}$ bonds are nearly covalent single bonds. However, their covalency degree is reinforced when going from compound $\mathbf{1}$ to $\mathbf{2}$ (see Table I). $\mathrm{f}\left(\mathrm{C}_{1} \mathrm{~N}_{2}\right)$ increases by increasing only the electron donating ability (case $\mathbf{2}$ and $\mathbf{3}$ ); it is worth noticing that the corresponding bond lengths are identical. The force constant values in the series (5.28 to $6.52 \mathrm{mdyn} \AA^{-1}$ ) suggest a trend to localize the $\pi$ electrons between the $-\mathrm{N}=\mathrm{CH}-$ double bond and the aniline phenyl ring. The interaction of the bridge nitrogen lone pair and the aromatic $\pi$ system should be essentially zero for a planar conformation. As a consequence, we expect the stretching mode $v\left(\mathrm{C}_{1} \mathrm{~N}_{2}\right)$ to be shifted to higher frequencies. This effect is verified for the absorption band at $1310 \mathrm{~cm}^{-1}$ in NBA which is observed at $1320 \mathrm{~cm}^{-1}$ and $1370 \mathrm{~cm}^{-1}$ in compounds 2 and 3, respectively (see Fig. 2). This result is in agreement with the observed frequencies and assignments proposed for $p$-aminoazobenzene derivatives [12], $p$-hydroxiazobenzene [13] and related compounds [14]. We should also expect to observe an increasing of the relative intensity of $v\left(\mathrm{C}_{1} \mathrm{~N}_{2}\right)$ bonds when increasing the bond polarity. Unfortunately the spectra are nearly impossible to analyse because of a significant coupling of $v\left(\mathrm{C}_{1} \mathrm{~N}_{2}\right)$ with other vibrational modes.

\section{$C_{3} C_{5}$ bonds}

Table I shows that the $\mathrm{C}_{3} \mathrm{C}_{5}$ single bond distance decreases by increasing the electron donating property of substituents. The polarity increasing in the same sense suggests an important charge redistribution effect. The observed trend of Wiberg indices allow us to predict a $\pi$-delocalization between the $-\mathrm{N}=\mathrm{CH}-$ double bond and the benzylidene ring which should be essentially maximum for a planar conformation but decreases when increasing the twisting angle $\varphi$ (see Fig. 1). Therefore, $f\left(C_{3} C_{5}\right)$ values are expected to increase with electron donor characteristics. The values indicate that a shifting to higher frequencies must be observed for the corresponding vibrational bands. We propose the absorptions at 1190,1190 and $1205 \mathrm{~cm}^{-1}$ of compounds $\mathbf{1 , 2}$ and $\mathbf{3}$, respectively, to be attributed to the stretching $\mathrm{C}_{3} \mathrm{C}_{5}$ vibration; the corresponding Raman intensity decreases in the same order (see Fig. 2). This assignment is in agreement with others proposed for $p$ methylbenzonitrile [15] and stilbene [16].

\section{$\mathrm{CH}$ bonds}

From an ab initio study [1] it has been proposed that one of the factors determining conformation of $\mathrm{N}$-benzylideneaniline involves the interaction of the ortho hydrogen on the aniline ring $\left(\mathrm{H}_{25}\right)$ and the azomethine proton $\left(\mathrm{H}_{4}\right)$. This interaction is repulsive in the planar conformation but is relieved by a twisting of the N-phenyl bond (see Fig. 1). Nevertheless our results do not permit any vibrational interpreta- 
tion of that effect: the Wiberg indices and the resulting force constants are practically identical in the three molecules (see Table I). The $v(\mathrm{CH})$ modes are expected to be observed beyond $2900 \mathrm{~cm}^{-1}$.

\section{A-ring CC bonds}

The Wiberg indices values for $\mathrm{CC}$ bonds in the aniline ring are characteristics of an intermediate single-double bond (see Table I). A more detailed analysis of these values allow us to infer that the $\pi$ electrons will be preferentially localized on the $\mathrm{C}_{16} \mathrm{C}_{17}\left(\mathrm{C}_{20} \mathrm{C}_{19}\right)$ bond when increasing electron donor and acceptor strengths. However, its adquired covalency degree remains almost invariant by changing only the electron donor groups. On the adjacent bonds a similar trend is verified. The resulting force constants are quite similar in the whole A-ring group. $\mathrm{f}\left(\mathrm{C}_{1} \mathrm{C}_{16}\right)$ decreases by increasing electron donor and acceptor abilities. Nevertheless the force constant for the central bond $\mathrm{C}_{16} \mathrm{C}_{17}\left(\mathrm{C}_{20} \mathrm{C}_{19}\right)$ increases exclusively when increasing the electron withdrawing characteristics; $\mathrm{f}\left(\mathrm{C}_{17} \mathrm{C}_{18}\right)$ decreases in the same manner. Thus, it can be inferred that the $\mathrm{f}\left(\mathrm{C}_{1} \mathrm{C}_{16}\right)$ variations are mainly due to the influence of electron donating groups.

\section{B-ring CC bonds}

Table I shows that the Wiberg indices values for the $\mathrm{C}_{6} \mathrm{C}_{7}\left(\mathrm{C}_{10} \mathrm{C}_{9}\right)$ bond increase with the electron donor strength. In the same sense the adjacent bonds become electron deficient. This result is well interpreted by the corresponding force constants: within the series of molecules $\mathrm{f}\left(\mathrm{C}_{6} \mathrm{C}_{7}\right)$ value increases by increasing the electron donating properties. Because of the difference between $\mathrm{f}\left(\mathrm{C}_{5} \mathrm{C}_{6}\right)$ or $\mathrm{f}\left(\mathrm{C}_{7} \mathrm{C}_{8}\right)$ and $\mathrm{f}\left(\mathrm{C}_{6} \mathrm{C}_{7}\right)$ with $\mathrm{f}(\mathrm{CC})$ values in the A-ring, we propose to assign the higher frequency to the $v\left(\mathrm{C}_{6} \mathrm{C}_{7}\right)$ mode in compound 3; this band is observed at $1600 \mathrm{~cm}^{-1}$ in absorption and at $1612 \mathrm{~cm}^{-1}$ in Raman (see Fig. 2). This assignment is consistent with the proposed ones for stilbene $\left(1594 \mathrm{~cm}^{-1}\right)$ [16] and anisole $\left(1588 \mathrm{~cm}^{-1}\right)$ [17]. The lowest frequency at $1320 \mathrm{~cm}^{-1}$ in the spectra of compound 3 could be ascribed to the $v\left(\mathrm{C}_{7} \mathrm{C}_{8}\right)$ mode (see Fig. 2). The same assignments have been proposed in stilbene $\left(1339 \mathrm{~cm}^{-1}\right)$ [16] and anisole $\left(1332 \mathrm{~cm}^{-1}\right)$ [17].

Infrared and Raman frequencies of the most relevant stretching vibrations in compounds $\mathbf{1}, \mathbf{2}$ and $\mathbf{3}$ are summarized in Table II.
Tab. II. Infrared (IR) and Raman frequencies $\left(\mathrm{cm}^{-1}\right)$ and proposed assignment for stretching modes in N-benzylideneaniline (1), $p$-methylbenzylidene-p-nitroaniline (2) and $p$-dimethylaminobenzylidene- $p$-nitroaniline $(\mathbf{3})$.

\begin{tabular}{lcrll}
\hline Compound & $v\left(\mathrm{~N}_{2} \mathrm{C}_{3}\right)$ & $v\left(\mathrm{C}_{1} \mathrm{~N}_{2}\right)$ & $v\left(\mathrm{C}_{3} \mathrm{C}_{5}\right)$ \\
\hline $\mathbf{1}$ & 1625 & 1310 & 1190 & IR \\
& 1627 & - & 1193 & Raman \\
$\mathbf{2}$ & 1620 & 1320 & 1190 & IR \\
& 1622 & 1318 & 1195 & Raman \\
$\mathbf{3}$ & 1575 & 1370 & 1205 & IR \\
& 1577 & - & 1209 & Raman \\
\hline
\end{tabular}

\section{Conclusions}

The calculated force constants, which are in good agreement with reported data [10], allowed us to propose a vibrational assignment of the most relevant stretching modes of three $p, p^{\prime}$-disubstituted $\mathrm{N}$-benzylideneanilines. The observed frequency shifting explains quite well the experimental conformational structure of these compounds. The trend to the planarity when increasing mainly the electron donor property could be explained on the basis of an intramolecular electron transfer between substituents. In fact, besides the stabilization of a nonplanar structure due to both an interaction of the bridge nitrogen lone pair and the aromatic $\pi$-system and a repulsion between the azomethine hydrogen and one of the ortho hydrogen on the aniline ring [1], delocalization of $\pi$-electrons between benzylidene and aniline rings through the $-\mathrm{N}=\mathrm{CH}-$ double bond plays an important role in stabilizing a less nonplanar structure. Similar conclusions have been obtained from a crystal structure analysis of two isomeric benzylideneaniline derivatives [7].

The authors would like to thank Dr. R. G. Morales for kindly supplying the molecules used in this study and to the Laboratoire de Spectroscopie Infrarouge at the Université de Bordeaux I for use of the Coderg T800 Raman spectrometer. This work was financially supported by grants No 310 from the FONDECYT and Q 2815-8812 from the Departamento Técnico de Investigación of the Universidad de Chile. We thank the SECI of Universidad de Chile for providing free computer time and Prof. R. Contreras R. for helpful discussion. 
[1] J. Bernstein, Y. M. Engel, and A. T. Hagler, J. Chem. Phys. 75, 2346 (1981); R. Akaba, K. Tokumaru, and T. Kobayashi, Bull. Chem. Soc. Jpn. 53, 2002 (1980); L. N. Patnaik and S. Das, Int. J. Quantum Chem. 27, 135 (1985).

[2] W. Kosmus, Z. Naturforsch. 33a, 1056 (1978).

[3] G. Diaz F., W. Kosmus, and K. Kalcher, Z. Naturforsch. 36a, 759 (1981); V. Vargas, E. Clavijo, and R. G. E. Morales, Z. Naturforsch. 40 a, 927 (1985).

[4] P. J. Mc Carty, R. J. Hovey, K. Veno, and A. Martell, J. Am. Chem. Soc. 77, 5820 (1955); G. Smets and A. Delvaux, Bull. Soc. Chim. Belg. 56, 106 (1947).

[5] J. A. Pople and D. Beveridge, Approximate Molecular Orbital Theory, Mc Graw Hill, New York (1970).

[6] H. B. Burgi and J. D. Dunitz, Chem. Commun. 1969, 472.

[7] H. Nakai, M. Shiro, K. Ezumi, S. Sakata, and T. Kubota, Acta Crystallogr. B32, 1827 (1976).

[8] K. W. Wiberg, Tetrahedron 24, 1083 (1968).
[9] R. Aroca and E. A. Robinson, J. Phys. Chem. 86, 894 (1982).

[10] E. B. Wilson, J. C. Decius, and P. C. Cross, Molecular Vibration, Mc Graw Hill, New York (1955).

[11] J. J. Lopez-Garriga, G. T. Babcock, and J. F. Harrison, J. Am. Chem. Soc. 108, 7241 (1986).

[12] K. Machida, B. K. Kim, Y. Saito, K. Igarashi, and T. Uno, Bull. Chem. Soc. Jpn. 47, 78 (1974).

[13] T. Uno, H. Lee, Y. Saito, and K. Machida, Spectrochim. Acta 32 A, 1319 (1976).

[14] R. N. Jones and C. Sandorfy, Techniques of Organic Chemistry, Vol. IX, Chemical Applications of Spectroscopy, Ed. W. West, Intersc. Publish. Inc. New York (1956).

[15] C. L. Chatterjee, P. P. Garg, and R. P. Jaiswal, Spectrochim. Acta 34 A, 943 (1978).

[16] Z. Meic and H. Gusten, Spectrochim. Acta 34A, 101 (1978).

[17] W. J. Balfour, Spectrochim. Acta 39 A, 795 (1981). 